\title{
A Flight Load Test Method for Helicopter Rotor Blade
}

\author{
Jiahong Zheng \\ Institute of Flight Test Technology, Chinese Flight Test Establishment, Xi'an, China
}

Email address:

040715316@163.com

\section{To cite this article:}

Jiahong Zheng. A Flight Load Test Method for Helicopter Rotor Blade. International Journal of Mechanical Engineering and Applications. Vol. 9, No. 5, 2021, pp. 75-78. doi: 10.11648/j.ijmea.20210905.11

Received: September 26, 2021; Accepted: October 18, 2021; Published: October 29, 2021

\begin{abstract}
This paper studies a flight load test method for helicopter blade, including the structural force of helicopter blade, strain gauge bridge modification, load calibration method and the establishment of load calibration equation in the typical maneuvers. First, blade structure of the helicopter was analyzed and the strain gauges were pasted in the load profile which were considered to be important positions to analysis the flight safe of helicopter. Second, Load calibration equations were obtained by ground calibration test, which would be used to convert the strain time curve to the load time curve. The helicopter flighted in different maneuvers, such as hovering, horizontal flight and climbing. At the same time, flight parameters were recorded, such as helicopter altitude, pitch angle, roll angle and yaw Angle. The flight parameters can be used to analysis the flight conditions. Then the blade flight load in different flight maneuvers was analyzed. The result was that the time-domain waveform was neat, good periodicity, strong regularity, no hybrid interference and jump point. It was illustrated that the blade load test flight data were accurate and reliable, which met the accuracy requirements of Engineering. The blade load data obtained through different flight maneuvers can be used to structural modification, fatigue analysis and blade design.
\end{abstract}

Keywords: Helicopter, Rotor Blade, Flight Test, Load Measurement

\section{Introduction}

The rotor blade is the main lift component of the helicopter, and it is also one of the main characteristics of the fixed wing. The helicopter rotor blade has the special aerodynamic environment and structural characteristics, as well as the resulting special aerodynamic, structural, and kinetic problems.

While the rotor blade rotates around the rotor axis, it also randomly moves together in different directions, and the aerodynamic environment is complex. The rotor blade also has three basic motion properties: swing motion (perpendicular to the rotor rotation surface), lag motion (within the rotation surface), and torsional motion around its own axis. The blades therefore produce swing, lag, and torsional loads during flight. All three loads cause fatigue damage at the roots of the blade, affecting the life of the blade roots. The study of blade swing load, lag load and torsion load has been one of the basic and important topics in the development of helicopter theory and technology, but also one of the complex and difficult problems [1].

Blade load analysis is typical of the analysis of aerodynamic and structural coupling. At present, many theoretical studies of blade load at home and abroad start from the root cause of blade load generation, and through repeated iteration between aerodynamic calculation and structural deformation calculation. First, a large number of aerodynamic is calculated to obtain the blade profile lift change and load distribution, and then the structural deformation is calculated to obtain the structural deformation of the blade under this load, and recalculates the aerodynamic power after deformation. This research method, which requires a precise aerodynamic model and more known conditions. Pneumatic analysis models, both from uniform inflow, predetermined wake, and free wake, despite increasingly precise loading calculations, have obvious deficiencies and limitations [2-8].

The helicopter rotor blade load flight test is mainly to use the flight test to measure the load of the helicopter rotor blade in the horizontal flight, hovering and other flight states. This paper studies the structural form of helicopter blade, strain gauge bridge modification, load calibration scheme and the establishment of load calibration equation in the typical flight test, and test data and results can be used for blade structural modification and life evaluation. 


\section{Blade Load Analysis}

When the helicopter rotor blades do not rotate, the blades are subjected to vertical downward gravity. As the rotor rotates, it is also subject to aerodynamic and inertial centrifugal forces. As shown in Figure 1, the result of the load action is to form swing, lag and torsional bending moments in each section of the blade. The aerodynamic tension moves up in the opposite direction of gravity, which circles the torque formed by the horizontal hinge to swing the rotor. The moment formed by the inertial centrifugal force relative to the horizontal hinge strives to rotate the blade in the rotation plane of the rotor hub. In the hovering or vertical flight state, the result of the three torque synthesis keeps the rotor blade in the rotor hub rotation plane at an angle, and the rotor forms an inverted cone. The typical force of a certain type of blade is shown in Figure 1.

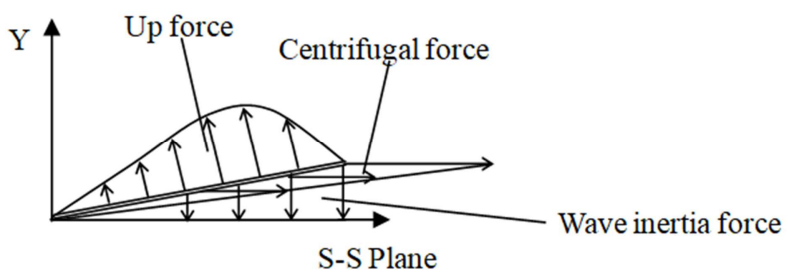

Figure 1. Diagram of blade force.

\section{Test Principle and Test Modification of Blade}

\subsection{Strain Measurement Technology}

(a) Principle of strain

The strain-resistance effect of the wire can directly transform the response variable of the component surface into the relative change amount of the resistance, so that the strain measurement can be measured by electricity. The strain gauge is the sensing element made by this principle. Before measuring, paste the strain gauge on the measured part of the component with an adhesive. Thus, the strain gauge can be deformed together with the measured part of the component. When the strain gauge elongates or shortens, the resistance changes. The relative resistance change of the strain gauge is proportional to its strain.

$$
\frac{\Delta R}{R}=K \frac{\Delta l}{l}=K \varepsilon
$$

Where, $K$ is sensitivity coefficient of the resistance strain gauge. $\frac{\Delta R}{R}$ is the relative resistance change of the strain gauge. $\varepsilon$ is strain.

(b) Bridge measurement circuit

When the strain is measured using the strain gauge, the strain gauge acts simply to convert the strain into a change in resistance. This change in resistance is usually measured using the Wheston Bridge. The strain gauge is used as an arm of the bridge. When the resistance value of the strain gauge changes, the output voltage of the bridge changes and the voltage change value is recorded or indicated for observation.

A full-bridge measurement circuit is used to convert small resistance changes of the strain gauge into a voltage signal, while maximizing the value of the strain. The full bridge measuring circuit is a resistance strain gauge on four bridge arms with a sensitivity coefficient, forming the full bridge. The output voltage can be obtained by formula (2).

$$
U_{0}=\frac{u}{4} k\left(\varepsilon_{1}-\varepsilon_{2}+\varepsilon_{3}-\varepsilon_{4}\right)=u k \varepsilon
$$

Then, the output strain can be obtained by formula (3) [9-11].

$$
\varepsilon=\frac{u_{0}}{k u}
$$

\subsection{Layout of Measuring Position of Blade Strain Gauge}

Seven key profiles were selected according to the theoretical blade stress distribution results, and strain gauges were pasted on the upper and lower surface of the blade to form a full bridge to measure the swing strain. Single gauge was attached to the leading and trailing edges of the upper surface of the blade to form a full bridge used to measure the lag strain. The upper surface and lower surface of the quarter axis of the blade are gauge symmetrically attached along $45^{\circ}$ degrees to form a full bridge to measure torque strain. The blade maximum stress area and dangerous stress area were affixed with strain gauges. The strain gauge position is shown in Figure 2.

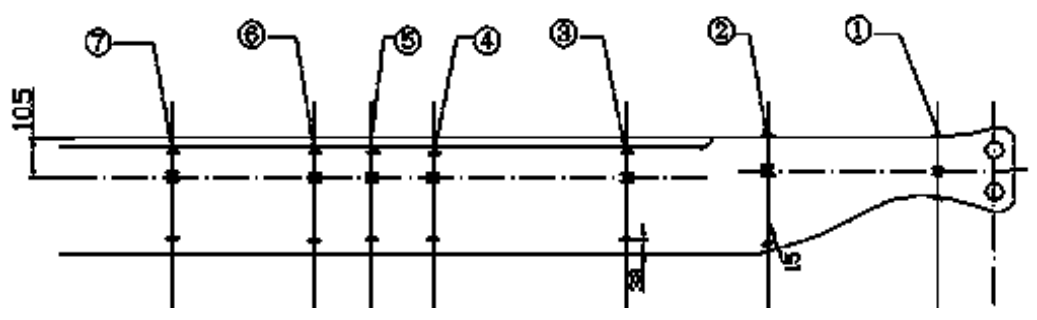

Figure 2. Schematic of main blade measurement.

\subsection{Load Calibration Test}

The blade load calibration equation is applied to the strain value measured by the helicopter to convert the strain time curve to the load time curve. This is the premise of strength design, reliability analysis and full-size fatigue test. The blade load calibration equation is a linear equation, since the blade stress under normal load is within the elastic range. 
Blades used for the test are used for the helicopter flight test. Joint calibration of the strain gauge together with the test equipment reduces the system error. During the calibration, the blade was first installed on the special calibration table. Preloading occurred before each load. The load was loaded in 5 stages and then unloaded to 0 after reaching the calibration load $[12,13]$.

Raw data for the load calibration test were obtained from the ground calibration test. Load calibration test data were treated with linear fitting to obtain the calibration equations for blade swing, lag and torsion. It was shown in Figure 3.

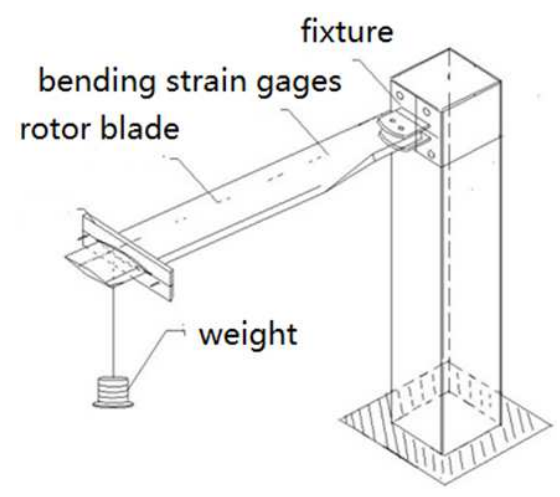

Figure 3. Blade load calibration.

To verify the accuracy of the blade load calibration equation, the strain value of each channel was measured by a static strain instrument through the load equation. The accuracy of the equation was tested by comparing the real values. It was proved that the relative error of test value and real value is less than $5 \%$, meeting the requirements of engineering accuracy.

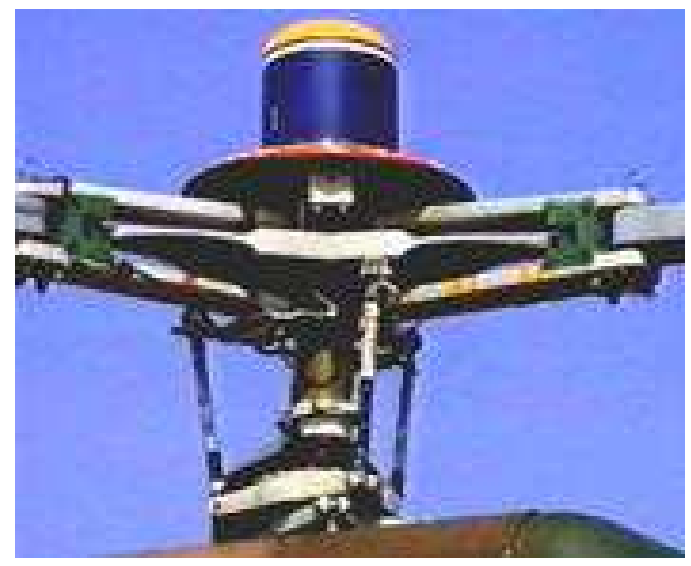

Figure 4. Rotor load test equipment

\subsection{The Test Equipment}

Rotor is a rotating part, and how to recorded strain signal without affecting flight safety is the difficulty of blade load measurement. The helicopter rotary part strain test equipment was designed to install at the top of the rotor hub with the rotor hub synchronous rotation. Strain measurement signal was recorded through wireless transmission. The helicopter simultaneously recorded flight parameters such as flight speed, altitude, acceleration and overload through the KAM500 device. GPS was used to keep the flying participation blade strain test equipment synchronized.

\subsection{Test Flight Method}

During the flight test, the helicopter flight in different maneuvers, such as hovering, horizontal flight and climbing. Flight parameters were recorded in flight test, such as helicopter altitude, pitch angle, roll angle and yaw Angle.

\section{Test Measurement Results and Analysis}

\subsection{Statistical Calculation}

For the measured load-time curve of each flight state of the helicopter, the load separation cycle was adopted to separate the load movement and static values. The load separation period is determined according to the base frequency period of the main load, and can usually be determined by the azimuthal signal of the rotor or tail hub. Load dynamic values and static values in each separation period were shown as follow $[14,15]$.

$$
\begin{aligned}
\text { Dynamic }_{\text {beep }} & =\frac{\text { Max }_{\text {beep }}-\text { Min }_{\text {beep }}}{2} \\
\text { Static }_{\text {beep }} & =\frac{\text { Max }_{\text {beep }}+\text { Min }_{\text {beep }}}{2}
\end{aligned}
$$

Where, Max ${ }_{\text {beep }}$ is the maximum load per cycle. Min $n_{\text {beep }}$ is the minimum load per cycle.

\subsection{Typical Flight Curve}

Figure 5 to Figure 7 shows the horizontal flight state blade load time curve of the helicopter at the normal center of gravity. It can be seen that the time-domain waveform was neat, good periodicity, strong regularity, no hybrid interference and jump point.

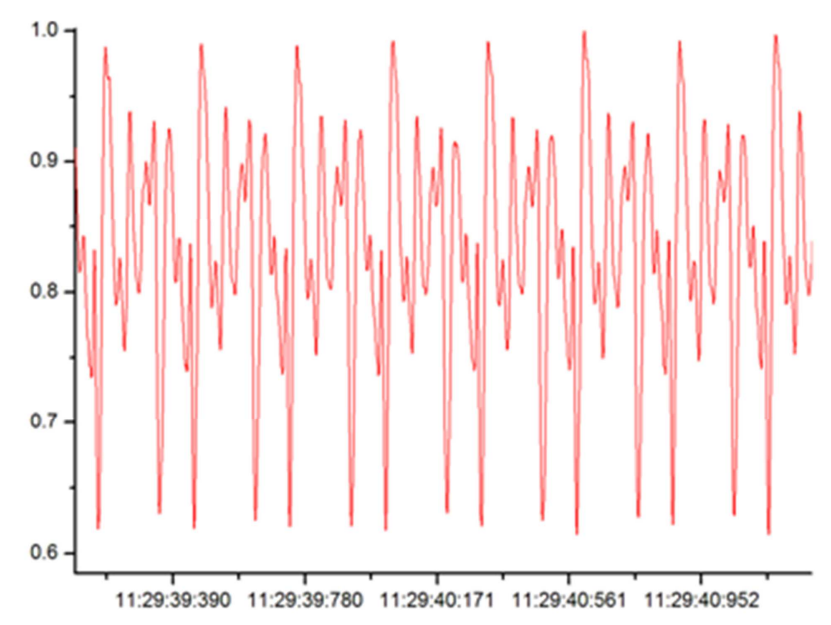

Figure 5. Blade swing load time curve in horizontal flight maneuver. 


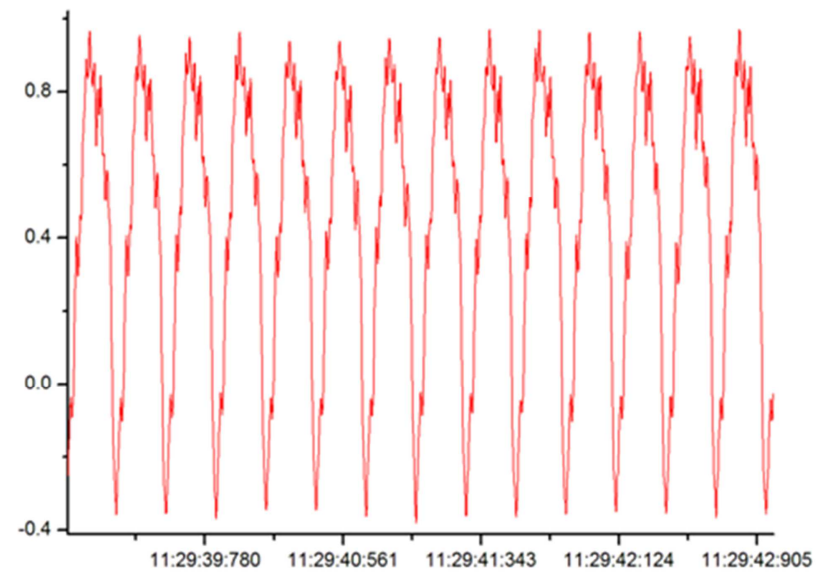

Figure 6. Blade lag load time curve in horizontal flight maneuver.

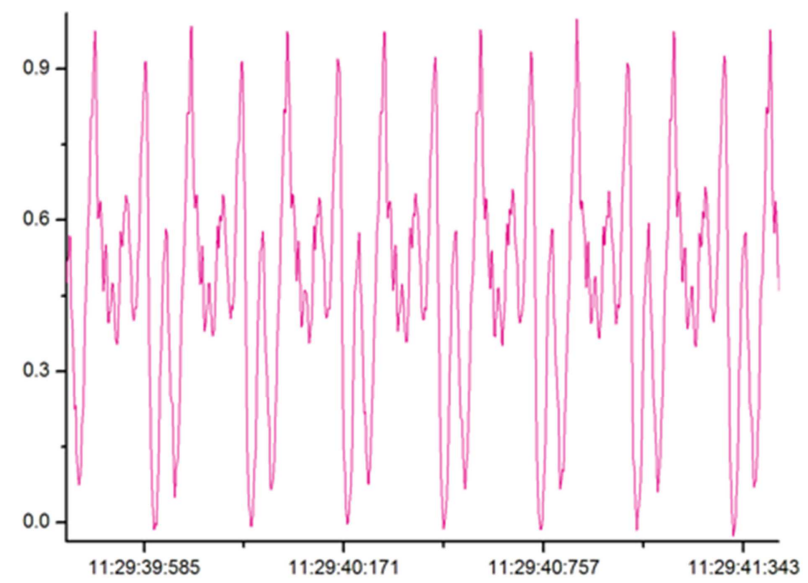

Figure 7. Blade torsion load time curve in horizontal flight maneuver.

\section{Conclusion}

This paper presents a flight load test method for helicopter blades. It analyzed the force of the helicopter blade structure. Then, through the ground calibration test and flight test, the blade flight load was obtained. The results showed that the helicopter blade load measurement scheme was feasible, accurate and reliable, which met the accuracy requirements. The blade load data obtained through different flight maneuvers can be used to structural modification, fatigue analysis.

\section{Future Work}

It is recommendation that the change law of the flight maneuvers, such as horizontal flight, climbing and hovering can be analyzed through the measured flight data.

\section{References}

[1] Sun Zhichao, Xiao qiuting, et al. Helicopter strength, aviation industry press, 1990.

[2] $\mathrm{Xu}$ Guohua, Wang Shicun. Calculation of free wake of helicopter rotor in forward flight [J]. Journal of Nanjing University of Aeronautics and Astronautics. 1997 (06).

[3] Lou Wuchen, Wang Shicun. Research progress of helicopter rotor wake [J]. Acta Aeronautica Sinica. 1990 (03): 113-119.

[4] Liu Mingming, Liu Bingkun, Dai Qiang. Helicopter rotor blade load measurement method [J]. Electronic technology and software engineering. 2020 (03): 97-99.

[5] Zhu Shijin, Li Nanhui. Calculation and analysis of helicopter rotor aerodynamic load [J]. Journal of Nanjing University of Aeronautics and Astronautics. 1979 (03): 98-113.

[6] Wang huanjin, Gao Zheng. Calculation method of dynamic aerodynamic load of helicopter rotor blade [J]. Journal of Nanjing University of Aeronautics and Astronautics. 1999 (01).

[7] Xu Guang, Wang Bo, xu Guohua, Zhao Qijun. Flow field and aerodynamic calculation of helicopter rotor based on CFD [J]. Journal of Nanjing University of Aeronautics and Astronautics. 2011 (03): 369-374.

[8] Wang Haowen, Gao Zheng. Calculation of rotor unsteady aerodynamic load using comprehensive aeroelastic analysis method [J]. Journal of Nanjing University of Aeronautics and Astronautics. 2003 (03): 268-272.

[9] Li Qiaozhen, Li Gang, Han Yinze. Experiment and application of resistance strain gauge [J]. Laboratory research and exploration, 2011 (4): 134-137.

[10] Liu Xiumei. Principle analysis and application discussion of resistance strain sensor [J]. Zhifu times. 2019 (03): 177.

[11] Li Ke. Measurement performance analysis of resistance strain sensor $[\mathrm{J}]$. Electronic components and information technology. 2020 (02): 153-155.

[12] Han Yuwang, Zhu Guangming, Chen Gongli. Study on a new method of helicopter rotor blade load calibration [J]. Helicopter technology. 2012 (03): 29-33.

[13] Liu Zhengjiang, Chen Huan, Hu Heping. Research on model blade calibration technology [J]. Helicopter technology, 2009 (3): 108-109.

[14] Li Yongshou, Tang Lifang. Research on load test of helicopter transmission system in flight $[\mathrm{J}]$. Introduction to scientific and technological innovation. 2011 (06): 2-3.

[15] Xi Juan, Wu Yuping, Chen Pingjian. Analysis and application of helicopter rotor load flight test results [J]. Journal of Nanjing University of Aeronautics and Astronautics. 2011 (03): 419-422. 